\title{
Could Machine Learning be used to address Africa's Challenges?
}

\author{
Noe Elisa \\ Department of Computer science \\ The University of Dodoma
}

\begin{abstract}
Machine Learning can be both experience and supervised based learning. Machine learning would help in designing system that can be able to take decisions in a more optimized form and also help them to work in most efficient method. In the field of machine learning one considers the important question of how to make machines able to "learn". Learning in this context is understood as inductive inference, where one observes examples that represent incomplete information about some "statistical phenomenon". Advanced economies are already using machine learning to solve problems like medical diagnosis, Improving Ecommerce Conversion Rates, traffic congestion, saving cows from bad drivers and improving healthcare, while Africa lags conspicuously behind. African leaders may be aware of this. Whether they possess the foresight to see and take people through is however debatable.
\end{abstract}

This paper discusses how machine learning could be used to address Africa's challenges by highlighting how some of major challenges can be solved using certain machine learning techniques. Major challenges to Africa continent are identified and machine learning techniques that could address them are briefly highlighted.

While it does have some frightening implications when thinking about it, machine learning applications are several of the many ways this technology can improve our lives.

\section{General Terms}

Africa's Challenges, Algorithm, Machine Learning.

\section{Keywords}

Africa's Challenges, Africa Continent, Algorithm, Machine Learning.

\section{INTRODUCTION}

Machine Learning (ML) is a technique that uses data analysis to automate and build analytical model. Using algorithms that iteratively learn from data, machine learning allows computers to find hidden insights without being explicitly programmed where to look [1]

Because of new computing technologies, machine learning today is not like machine learning of the past. It was born from pattern recognition and the theory that computers can learn without being programmed to perform specific tasks; researchers interested in artificial intelligence wanted to see if computers could learn from data. The iterative aspect of machine learning is important because as models are exposed to new data, they are able to independently adapt. They learn from previous computations to produce reliable, repeatable decisions and results. It's a science that's not new but one that's gaining fresh momentum. Today, machine learning algorithms are being used to crunch massive amounts of information from many different sources and provide insights on everything from patient care decisions in clinical medicine to pedestrian recognition in driverless cars and managing renewable energy within a power grid [2].

While many machine learning algorithms have been around for a long time, the ability to automatically apply complex mathematical calculations to big data over and over, faster and faster is a recent development.

The following are few widely publicized examples of machine learning applications most people may be familiar with:

The heavily advertised, self-driving car by Google. The essence of machine learning based on deep leaning.

Online recommendation offers such as those from Amazon and Netflix are machine learning applications for everyday life.

Knowing what customers are saying about different events can realized on Twitter. Machine learning combined with linguistic rule creation.

Machine learning for fraud detection is one of the more obvious, important uses in our everyday lives.

After a brief discussion on machine learning, it is time to discuss about africa's challenges and propose how machine learning will help in combating these problems.

Africa is the world's second-largest and second-mostpopulous continent. It hosts a large diversity of ethnicities, cultures and languages. All of these features made the continent face the hardest challenges in the world. Top challenges facing Africa today include but not limited to Poverty, Poor Education, Poor Health Systems, Violence, Hunger, Sustainable agriculture, nutrition and food security, Access to financing and as well Economic growth rate is far too low.

The main goal of this paper is to highlight how Machine Learning (ML) can be used to address Africa's Challenges. The next section cover a brief background on Africa continent and machine learning, section 3.0 covers discussion and finally conclusion is presented.

\section{BACKGROUND}

This section discusses Africa continent in brief and then introduces machine learning classes with their associated algorithms that can be used in developing learning models.

\subsection{Africa Continent}

Africa is the World's second-largest continent with $30,065,000$ square $\mathrm{km}$, covering approximately $20 \%$ of the Earth's land and $6 \%$ of the Earth's surface. It is bordered by the Atlantic Ocean to the east, the Indian Ocean to the west 
and the Mediterranean Sea to the north. The Red Sea and Suez Canal lie to the north-east and separate Africa from Asia.

The longest river in the World, The Nile, measuring $6,695 \mathrm{~km}$ flows north ending in a delta that empties into the Mediterranean Sea [3]. The World's largest desert, The Sahara, measuring 9,000,000 $\mathrm{km}^{2}$, covers much of north Africa. The Kalahari desert in south-west Africa measures 259,000 square $\mathrm{km}$. The Atlas mountain range lies in the north-west of Africa and the highest mountain, Kilimanjaro, $5895 \mathrm{~m}$, is in Tanzania.

The climate of Africa is governed by its position on the globe and can be broadly divided into five different climate types [4]:

Rainforest: This region is characterised by very high temperatures and high rainfall throughout the year.

Savanna: This region has very high temperatures all year and rain during the summer season only.

Steppe: This region has high temperatures all year and only limited rainfall during the summer season.

Desert: High temperatures throughout the year with very little rainfall.

Mediterranean: Warm to high temperatures with rainfall in the autumn and winter months.

The population of Africa is growing rapidly with a growth rate of approximately $3 \%$ per annum. The total population of Africa is approximately 1.2 billion [5] people as of 2016, it accounts for about $16 \%$ of the world's human population.

Despite of these beautiful natural resources in Africa there are a lots of challenges such as current economic growth rate is far too low, industrial development has been stalled since the 1970s, the lives of most Africans are marred by poverty, hunger, poor education, ill health, and violence. Whether hidden or obvious, malnutrition is arguably Africa's biggest impediment to sustained, equitable economic growth. While one in nine people in the world today is malnourished, in subSaharan Africa, that ratio is one in four. Malnourished children lag up to four years behind their peers in education achievement. In countries with persistently high levels of malnutrition, the economic costs can rise to 16.5 percent of GDP [5].

\subsection{Machine Learning}

Machine Learning field evolved from the broad field of Artificial Intelligence, which aims to mimic intelligent abilities of humans by machines. In the field of Machine Learning one considers the important question of how to make machines able to "learn". Learning in this context is understood as inductive inference, where one observes examples that represent incomplete information about some "statistical phenomenon Learning is considered as a parameter for intelligent machines" [6].

Deep understanding would help in taking decisions in a more optimized form and also help then to work in most efficient method. Machine learning systems automatically learn programs from data. This is often a very attractive alternative to manually constructing them, and in the last decade the use of machine learning has spread rapidly throughout computer science and beyond. Machine learning is used in Web search, spam filters, recommender systems, ad placement, credit scoring, fraud detection, stock trading, drug design, and many other applications [6].
Machine Learning can be both experience and explanation based learning. In the field of robotics machine learning plays a vital role, it helps in taking an optimized decision for the machine which eventually increases the efficiency of the machine and more organized way of preforming a particular task [7]. Now-a-days the concept of machine learning is used in many applications and is a core concept for intelligent systems which leads to the introduction innovative technology and more advance concepts of artificial thinking.

Different algorithms are introduced for different types of machines and the decisions taken by them. Designing the algorithm and using it in most appropriate way is the real challenge for the developers and scientists.

Machine learning is divided into supervised and unsupervised learning [8]. In Supervised Machine Learning, the process of an algorithm learning from the training dataset can be thought of as a teacher supervising the learning process. We know the correct answers, the algorithm iteratively makes predictions on the training data and is corrected by the teacher. Learning stops when the algorithm achieves an acceptable level of performance. Supervised learning involves classification whereby the output variable is a category, such as "red" or "blue" or "disease" and "no disease".

Unsupervised Machine Learning unlike supervised learning above there is no correct answers and there is no teacher. Algorithms are left to their own devises to discover and present the interesting structure in the data. Unsupervised learning problems can be further grouped into clustering and association problems. A clustering problem is where you want to discover the inherent groupings in the data, such as grouping customers by purchasing behavior. An association rule learning problem is where you want to discover rules that describe large portions of your data, such as people that buy $\mathrm{X}$ also tend to buy Y. Semi-Supervised Machine Learning problems arise when you have a large amount of input data $(\mathrm{X})$ and only some of the data is labeled (Y). These problems sit in between both supervised and unsupervised learning.

Some of machine learning algorithms used in problem solving are:

Decision Trees: Decision tree is a type of supervised learning algorithm for classification problems. It works for both categorical/classified and continuous input and output variables. In this technique, the population or sample are split into two or more homogeneous sets (or sub-populations) based on most significant differentiator in input variables.

Support Vector Machine: It is used for classification problems. The algorithm plot each data item as a point in ndimensional space (where $\mathrm{n}$ is number of features you have) with the value of each feature being the value of a particular coordinate.

Naive Bayes: Is a classification technique based on Bayes' theorem with an assumption of independence between predictors. In simple terms, a Naive Bayes classifier assumes that the presence of a particular feature in a class is unrelated to the presence of any other feature. For example, a fruit may be considered to be an apple if it is red, round, and about 3 inches in diameter.

K- Nearest Neighbors: Is a simple machine learning algorithm that stores all available cases and classifies new cases by a majority vote of its $\mathrm{k}$ neighbors. The case being assigned to the class is most common amongst its $\mathrm{K}$ nearest neighbors measured by a distance function. 
These distance functions can be Euclidean, Manhattan, Minkowski and Hamming distance.

K-Means: Is an unsupervised machine learning algorithm which solves the clustering problem. Its procedure follows a simple and easy way to classify a given data set through a certain number of clusters (assume $\mathrm{k}$ clusters). Data points inside a cluster are homogeneous and heterogeneous to peer groups.

Random Forest: In Random Forest, we've collection of decision trees (so known as "Forest"). To classify a new object based on attributes, each tree gives a classification and we say the tree "votes" for that class. The forest chooses the classification having the most votes (over all the trees in the forest).

Fuzzy c-means: is a method of clustering which allows one piece of data to belong to two or more clusters.

Artificial Neural Networks: are the biologically inspired simulations performed on the computer to perform certain specific tasks like clustering, classification, pattern recognition etc.

Deep Learning: Deep Learning has enabled many practical applications of Machine Learning and by extension the overall field of AI. Deep Learning breaks down tasks in ways that makes all kinds of machine assists seem possible, even likely. Driverless cars, better preventive healthcare, even better movie recommendations, are all here today or on the horizon.

\section{DISCUSSION}

In this section, major challenges facing Africa continent are discussed and propose how machine learning can be applied to combat them.

In sustainable agriculture, machine learning algorithm can be used to analyze weather and crop data. This will reveal drought on the horizon, and farmers will be advised to skip a planting season, saving them a lot of million US dollars in input costs. The weather report system with the help of machine learning would enable governments, donors, farmers, relief agencies, NGOs and others to implement more rapid, tailored interventions to prevent food crises. More specifically, the technology has the capability of forecasting underperforming crops in African countries and situations that will call for an international convention.

In Healthcare, a majority of African countries still have fewer than one doctor for every one thousand people, and with the African population expected reach 1.6 billion by 2030, up from 1 billion in 2010, this is not just problematic, but catastrophic [9]. Just as alarming is that the World Health Organization projects that by 2020 the burden of disease for chronic conditions like cancer, cardiovascular and respiratory disease, which all require specialist care, will far surpass that of infectious diseases. While this is a global trend, the impact on Africa will be worse because the continent has a much weaker healthcare system infrastructure, a short supply of equipment and medication, and a limited number of specialists for diagnosis and care. The easy answer is to build more hospitals and train more doctors, but this isn't enough. Africa needs to rise to this challenge and take a global leadership role in effectively merging healthcare and technology for care delivery and the most obvious answer to achieve this is through machine learning for prediction and analysis and Artificial Intelligence (AI) to develop a proper technology.
In fighting poverty, an approach that combines machine learning with high-resolution satellite imagery to provide new data on socioeconomic indicators of poverty and wealth could be used. Accurate information about people's needs could influence decisions about where to send aid or build roads or hospitals. In August, Stanford researchers published a paper on using satellite imaging and machine learning to track and measure poverty throughout Africa [10].Accurate measurement of poverty in Africa was extremely lacking as "39 out of 59 African countries conducted fewer than two surveys to measure poverty" between 2000 and 2010, according to The World Bank.

In African education, the UN has estimated that across the world more people have access to mobile phones than to toilets [5].It is of course distressing to imagine what this means for many people's exposure to education, disease and access to clean water, but the choice of mobile phone for the comparative statistic actually offers a great deal of hope. The mobile phone is part of a phenomenon where a new infrastructure is emerging, one that could bring the economic changes that enable people learn differently, at different speeds and with different starting points. Machine learning and Artificial intelligence could help in a future where we all learn in a much more personalised way. But no education system in the world can afford a tutor for every child, so this is where ML and AI might be able to step in. Artificial tutors, made to look and sound as much like humans as possible, could take the lead in delivering personalised education in Africa.

Food security of nations in Africa. Computer vision as part of machine learning can be used to detect plants diseases using images captured by low-cost mobile phones [11]. New data derived from satellites, insurance records, social media, and other sources can help Africans better understand agriculture and food security. By applying machine learning and computer models to data collected from high-resolution satellite imagery, researchers and policymakers will have access to poverty statistics that previously would have taken too much time or money to collect

Human immunodeficiency virus infection and acquired immune deficiency syndrome (HIV/AIDS) is one of the major burdens of disease in Africa and kill a number of people every year, and the standard-of-care treatment includes prescribing antiretroviral drugs. However, antiretroviral drug resistance is inevitable due to selective pressure associated with the high mutation rate of HIV. Determining antiretroviral resistance can be done by phenotypic laboratory tests or by computerbased interpretation algorithms. Additionally, machine learning can be used to shorten response times in mobile question-answering services, allowing these services to reach more people according to [12].Machine learning can extract valuable insights from unstructured data like clinical notes and academic journals to provide even larger datasets that will transform the medical industry into a proactive front against diseases.

There is no single health system in any country in Africa collecting, tracking and processing the many different indicators of malnutrition, which deprives decision-makers of critical insights that could drive more effective solutions despite of Africa still suffering from malnutrition. Signs of malnutrition may not become apparent until a food crisis erupts. Whether hidden or obvious, malnutrition is arguably Africa's biggest impediment to sustained, equitable economic growth. It can be difficult to detect the subtle factors that inevitably produce food shortages and chronic malnutrition 
before conditions degrade and hunger sets in. Using machine learning, it can be easy to predict climate changes and in turn help to foreseen food shortages eventually combat malnutrition.

Extending access in financial services with machine learning is possible in Africa. Machine learning could be used to create an intelligent risk credit scoring that has the potential to bring sustainable lending where banks in Africa have been too cautious in the past given lack of either collateral or credit history. Data becomes Africa's newest asset for fostering economic growth. Present use cases of machine learning in finance includes algorithmic trading, portfolio management, fraud detection and loan underwriting.

Can we use data and machine learning to predict violence in Africa? Yes. With ML, forecasting models and new data from communities can be used to predict outbreaks of local violence with high sensitivity and moderate accuracy, even with limited data. As well, an approach that combines machine learning with high-resolution satellite imagery and computer vision to provide new data on existence of potential violence can be developed. Machine learning in helping wildlife for instance can be used to create a technology for tracking animal movements, to see where they go, and what habitats we need to protect. By using computing power, machine learning can be used to figure out the best places to create wildlife corridors for certain kind of animals such as Rhinoceros, Elephant and the like.

While we wait for government policy to catch up, it's not too early for young innovators to set the ball rolling. In fairness, many are already taking the necessary baby steps. In Nigeria, for example, there are products like Lara.ng and Kudi.ai which bring a conversational twist to bus directions and bill payments respectively. But there is still much more that can be done with ML and data to solve Africa's existential problems.

For instance, African countries may be coming late to the information revolution, but they will be able to exploit the lessons learned from those that have trodden the path before. The UK's attempt to centralise its health system was an expensive failure [13].African countries don't now need to spend $£ 10$ bn to learn the lessons derived from that misguided effort. They could develop infrastructures for commerce, administration and health that exploit all the advantages of a distributed data system. African leaders are aware of this. Whether they possess the foresight to see people through is however debatable. Advanced economies are already using machine learning to solve problems like traffic congestion, saving cows from bad drivers and improving healthcare, while Africa lags conspicuously behind.

\section{CONCLUSION}

Because of new computing technologies, machine learning today is not like machine learning of the past. It was born from pattern recognition and the theory that computers can learn without being programmed to perform specific tasks; researchers interested in artificial intelligence wanted to see if computers could learn from data. The iterative aspect of machine learning is important because as models are exposed to new data, they are able to independently adapt. They learn from previous computations to produce reliable, repeatable decisions and results. Accurate measurements of economic characteristics are essential to shaping government and international development organizations' decisions on where to send aid. These measurements inform global efforts to understand and track progress towards improving human livelihoods

This paper highlights how machine learning could be used to solve challenges that African continent is facing. Each of identified challenge is discussed and a machine learning technique that can be used to address it is highlighted. Advanced economies are already using machine learning to solve problems like traffic congestion, saving cows from bad drivers and improving healthcare, while Africa lags conspicuously behind. African leaders may be aware of this. Whether they possess the foresight to see people through is however debatable.

\section{ACKNOWLEDGMENTS}

The author acknowledge the University of Dodoma, College of Informatics and Virtual Education for all the supports he received to allow this study to be undertaken.

\section{REFERENCES}

[1] J. Wen, S. Li, Z. Lin, Y. Hu, and C. Huang, "Systematic literature review of machine learning based software development effort estimation models," Inf. Softw. Technol., vol. 54, no. 1, pp. 41-59, 2012.

[2] G. Acampora, D. J. Cook, P. Rashidi, and A. V Vasilakos, "A Survey on Ambient Intelligence in Health Care.," Proc. IEEE. Inst. Electr. Electron. Eng., vol. 101, no. 12, pp. 2470-2494, 2013.

[3] B. Freund, "Africanist History and the History of Africa," Mak. Contemp. Africa, pp. 1-2, 1984.

[4] Y. Luan, X. Cui, and M. Ferrat, "Historical trends of food self-sufficiency in Africa," Food Secur., vol. 5, no. 3, pp. 393-405, 2013.

[5] P. D. United Nations, Department of Economic and Social Affairs, "World Population Prospects The 2017 Revision Key Findings and Advance Tables," World Popul. Prospect. 2017, pp. 1-46, 2017.

[6] S. B. Kotsiantis, "Supervised Machine Learning: A Review of Classification Techniques," Informatica, vol. 31, pp. 249-268, 2007.

[7] J. Qiu, Q. Wu, G. Ding, Y. Xu, and S. Feng, "A survey of machine learning for big data processing," EURASIP J. Adv. Signal Process., vol. 2016, no. 1, p. 67, 2016.

[8] S. B. Kotsiantis, I. D. Zaharakis, and P. E. Pintelas, "Machine learning: A review of classification and combining techniques," Artif. Intell. Rev., vol. 26, no. 3, pp. 159-190, 2006.

[9] AfDB, "Briefing Notes for AfDB's Long -Term Strategy. Briefing Note 6: Inclusive Growth Agenda," no. April, 2012.

[10] N. Jean, M. Burke, M. Xie, W. M. Davis, D. B. Lobell, and S. Ermon, "Combining satellite imagery and machine learning to predict poverty," Science (80-. )., vol. 353, no. 6301, pp. 790-794, 2016.

[11] J. Quinn and K. Leyton-Brown, "Modeling and Monitoring Crop Disease in Developing Countries,' Twenty-Fifth AAAI Conf., vol. 1, pp. 1390-1395, 2011.

[12] UNICE, "https://www.newscientist.com/article/2083044ai-helps-answer-thousands-of-health-queries-in-zambiavia-sms/." 2017.

[13] THEGUARDIAN,"https://www.theguardian.com/society /2013/sep/18/nhs-records-system-10bn.” 2017. 